\title{
Corneal Epithelial Oxygen Uptake Rate in Diabetes Mellitus
}

\author{
M. P. RUBINSTEIN, $\dagger$ S. T. PARRISH, ${ }^{*}$ S. A. VERNON $\dagger$ \\ Nottingham and Manchester
}

\begin{abstract}
Summary
Corneal epithelial oxygen uptake rate (CEOUR) has been measured using a noninvasive polarographic electrode technique in patients with diabetes mellitus (DM), all with varying degrees of retinopathy. CEOUR was found to be significantly reduced in diabetic patients when compared to age-matched non-diabetic controlsType I diabetics $p<0.001$; Type 2 diabetics $p<0.01$. Known duration of DM and assay of instantaneous, medium and long term glycaemic control showed no significant correlation with CEOUR in the patients studied. These results give further evidence of abnormal corneal metabolism in DM. Reduced CEOUR may be a contributory factor in the pathogenesis of diabetic corneal epitheliopathy.
\end{abstract}

Diabetes mellitus (DM) has various manifestations in the anterior segment of the eye. ${ }^{1,2}$ Within the cornea a variety of changes have been noted, for example reduced sensitivity, ${ }^{3}$ delayed epithelial healing, ${ }^{4}$ increased fragility of the epithelium ${ }^{5}$ and alterations in endothelial morphology. ${ }^{6}$ These phenomena have generally been explained by considering the modified metabolism of the tissue. ${ }^{6,7}$

Measurement of corneal epithelial oxygen uptake rate (CEOUR) gives an indication of the respiration characteristics of the cornea ${ }^{8}$ and it may be quite easily assessed in vivo by an applanation technique using a polarographic electrode. Although CEOUR has been shown to be abnormal or modified in various pathological states of the cornea ${ }^{9,10,11}$ it has received scant attention in DM. One laboratory study ${ }^{12}$ has indicated a decline in whole oxygen consumption in human and rat corneae. The present study was set up in order to measure CEOUR in diabetic patients and to assess any differences between Type 1 and Type 2 diabetics. Any possible correlation between CEOUR, duration of disease and state of glycaemic control was also monitored.

\section{Materials and Methods}

Patient acquisition and classification protocol Patients were recruited into the study from one ophthalmic diabetic clinic after informed consent and were placed into categories; Type 1 or Type 2 depending on known age of onset and mode of control of diabetes, according to contemporary classification protocol. ${ }^{13}$

Twenty-five patients were recruited into each of two groups coded 1 or 2 , according to type of diabetes. Group 1 consisted of 13 males and 12 females, age range $39.6 \pm 11.0$ years; the majority of patients in this Group had proliferative retinopathy. Group 2 consisted of 13 males and 12 females, age range $59.2 \pm 10.4$ years; patients in this group had a mixture of background and proliferative retinopathy. For the purposes of this study the extent, type or treatment of retinopathy was not considered, as these factors were considered to be too variable. Two groups of age- 
matched control subjects were also recruited consisting in the first group of 13 males and 12 females, age range $39.72 \pm 6.38$ years and in the second group, 13 males and 12 females, age range $58.03 \pm 9.2$ years. Diabetes was excluded in these control groups by routine urinalysis. Exclusion criteria from the study were previous ocular surgery, contact lens wear, concurrent use of eye medication, history of previous corneal pathology and evidence of corneal scarring.

\section{CEOUR measurement technique}

CEOUR was measured on both eyes of all subjects using a micro-polarographic electrode and monitor designed by one of the authors (STP) and previously described. ${ }^{14}$ The electrode was applied directly to the cornea after application of local anaesthetic drops (Ophthaine-proxymetacaine hydrochloride $0.5 \%$ ) and the sensor current amplified and linked to a microcomputer. The electrode was calibrated periodically at $34^{\circ} \mathrm{C}$ in air $\left(155 \mathrm{mmHg} \mathrm{pO}_{2}\right)$. The CEOUR was determined by measuring the fall in oxygen tension at the electrode membrane during a five second period of corneal applanation. Three readings of the CEOUR were recorded from the central zone of each cornea and the mean calculated for each eye.

\section{Blood analysis}

In the diabetic patients the known duration of the disease was recorded and a sample of venous blood taken for analysis. Blood analysis consisted of measurement of blood glucose, fructosamine and $\mathrm{HbA} 1$ to give indications of instantaneous, medium term and longer term glycaemic control respectively.

\section{Reliability of technique}

To illustrate the reliability of the micro-polarographic electrode used in this study a trialre-trial repeatability measurement was made on both eyes of a further 10 normal volunteer subjects. The interval between repeated measurements was one week.

\section{Results}

The result of testing reliability of the micropolarographic electrode showed a high corre- lation between repeated measurements (Pearson rho $=0.9)$ indicating good repeatability. A paired Students $t$ test was carried out on the results showing a non-significant difference between repeated measurements. In the analysis of clinical data from patients studied, the results from the right eye only were considered. The mean values of CEOUR for each diabetic and control group are shown in Table I. Using unpaired Students $t$ tests it was found tht there was a statistically significant lower CEOUR in both groups of diabetic patients when compared with their respective control group: in Group 1 $\mathrm{p}<0.001$, in Group $2 \mathrm{p}<0.01$.

Correlations between CEOUR and the assays of glycaemic control were found to be very poor. Considering blood glucose, fructosamine and HbA1 respectively, Pearson rho was calculated to be $0.22,-0.34$ and 0.34 for Group 1 and $-0.10,-0.26$ and -0.04 respectively. Correlation between duration of diabetes and CEOUR was also found to be poor: for Group 1 Pearson rho $=0.20$, for Group 2 Pearson rho $=-0.048$.

\section{Discussion}

The results of this study show that the CEOUR in patients with diabetic retinopathy is significantly lower than that of non-diabetic age-matched controls. The standard deviation for CEOUR in both groups of diabetic patients is higher than their respective control group and this probably reflects inter-patient variations in blood sugar levels. This variation in blood sugar levels is confirmed by poor correlation of our assay of glycaemic control with CEOUR. Statistically, the difference in CEOUR between diabetic patients and controls is greater for Type 1 and Type 2 diabetics. This is likely to be due to the fact that the majority of patients in Group 1 had prolifer-

Table I Mean values of CEOUR in Type 1 and Type 2 diabetic patients and their respective control groups. CEOUR measured in $\mu \mathrm{L} \mathrm{O}_{2} \mathrm{~cm}^{-2} \mathrm{hr}^{-1}$.

\begin{tabular}{lc}
\hline Patient group & CEOUR recorded \\
\hline & Mean SD \\
Type 1 diabetic & $4.40 \pm 0.99$ \\
Control group 1 & $5.50 \pm 0.59$ \\
Type 2 diabetic & $4.85 \pm 0.93$ \\
Control group 2 & $5.20 \pm 0.59$ \\
\hline
\end{tabular}


ative retinopathy and hence a more compromised ocular function than patients in Group 2.

Differences in CEOUR are primarily caused by differences in the metabolic activity of the corneal epithelium ${ }^{13}$ and our results thus indicate that the corneal epithelium in diabetics with retinopathy has a depressed rate of metabolism. This finding is in agreement with a previous study ${ }^{12}$ which considered oxygen consumption of in vitro normal and diabetic rat and human corneae.

This depressed metabolic rate of the corneal epithelium might be an aetiologic factor in the entity 'diabetic keratopathy'. ${ }^{15}$ The pathogenesis is far from clear but alterations in the sorbitol pathway have been implicated. ${ }^{4,7,16}$ Another common feature of the diabetic cornea is a reduced sensitivity., CEOUR measured in patients who have undergone penetrating keratoplasty has been shown to be reduced ${ }^{11}$ due to loss of neural integrity and similar observations have been in post-cataract surgery patients, depending on the degree of corneal denervation. ${ }^{10}$ It may be that the results of the present study can be partly explained by reduced corneal sensitivity, a factor not investigated in the study.

Measurement of conjunctival oxygen tension in diabetic patients with and without retinopathy ${ }^{17}$ has shown that the conjunctiva is likely to become increasingly hypoxic with increasing severity of diabetic retinopathy. Initial trials of measuring conjunctival oxygen uptake rate using our equipment proved to be extremely variable even in normal subjects depending on the degree of conjunctival hyperaemia following instillation of local anaesthetic drops.

Duration of diabetes and indicators of glycaemic control did not correlate significantly with CEOUR in our study and we are therefore not able fully to explain the findings.

The numerous anomalies of the corneal epithelium that have been reported in diabetic patients are likely to be due in part to cellular biochemical alterations and also a reduced corneal sensitivity. We suggest that a reduced metabolic rate (as measured by a reduced CEOUR) may be a further contrib- utory factor in the aetiology of diabetic keratopathy.

The authors gratefully acknowledge the financial support of Dollond and Aitchison Ltd.

References

${ }^{1}$ Kanksi JJ: Clinical Ophthalmology London: Butterworths, 1984, 38.

${ }^{2}$ Kritzinger EE and Wright BE: The eye in systemic disease. Chicago. Year Book Medical Publishers. 1984, 44.

${ }^{3}$ Macrae SM, Engerman RL, Hatchell DL: Corneal sensitivity and control of diabetes. Cornea 1982, 1: 223-6.

${ }^{4}$ Foulkes GN, Thoft RA, Perry HD, Tolentino FI: Factors related to corneal epithelial complications after closed vitrectomy in diabetics. Arch Ophthalmol 1979, 97: 1076-8.

${ }^{5}$ O'Leary DJ and Millodot M: Abnormal epithelial fragility in diabetes and contact lens wear. Acta Ophthalmol 1981, 59: 827-33.

${ }^{6}$ Schultz RO, Matsuda M, Yee RW, Edelhauser HF, Schultz KJ: Corneal endothelial changes in Type I and Type II diabetes mellitus. Am J Ophthalmol 1984, 98: 401-10.

${ }^{7}$ Kinoshita JH: Aldose reductase in the diabetic eye XLIII Edward Jackson Memorial Lecture. Am J Ophthalmol 1986, 102: 685-92.

${ }^{8}$ Larke JR: The eye in contact lens wear. London: Butterworths, 1985, 71.

${ }^{9}$ Hill RM and Lowther GE: Oxygen flux across the tear-epithelial interface as an index of corneal wound repair. Am J Optom 1967, 44: 267-75.

${ }^{10}$ Holden BA, Polse KA, Fonn D, Mertz GW: Effects of cataract surgery on corneal function. Invest Ophthal Vis Sci 1982, 22: 343-50.

${ }^{11}$ Vannas A, Holden BA, Sweeney DF: Epithelial metabolism of the corneal graft is abnormal. $\mathrm{Br} \mathrm{J}$ Ophthalmol 1987, 71: 593-7.

${ }^{12}$ Graham CR, Richards RD, Varma SD: Oxygen consumption by normal and diabetic rat and human corneas. Ophthal Res 1981, 13: 65-71.

${ }^{13}$ Alberti KG and Hockaday TD: Diabetes mellitus In: Weatherall DJ, Ledingham JGG and Warrell DA (eds) Oxford Textbook of Medicine. Oxford Medical Publications, Oxford. 1983: 9.5-9.49.

${ }^{14}$ Larke JR, Parrish ST, Wigham CG: Oxygen sensors for clinical use. J Br Contact Lens Assn 1980, 3: $70-4$.

${ }^{15}$ Schultz RO, Peters M, Sobocinski N, Nassif K, Schultz KJ: Diabetic keratopathy as a manifestation of peripheral neuropathy. Am J Ophthalmol 1983, 96: 368-71.

${ }^{16}$ Ohashi Y, Matsuda M, Hosotani H, Tano Y, Ishimoto I, Fukada M, Manabe R: Aldose Reductose Inhibitor (CT-112) Eyedrops for diabetic corneal epitheliopathy. Am J Ophthalmol 1988, 105: 233-8.

${ }^{17}$ Isenberg SJ, McRee WE, Jedrzynski MS: Conjunctival hypoxia in diabetes mellitus. Invest Ophthalmol Vis Sci 1981, 21: 317-21. 